\title{
Energy-Efficiency of Dual-Switched Branch Diversity Receiver in Wireless Sensor Networks
}

\author{
Ghaida A. AL-Suhail \\ Department of Computer Engineering \\ College of Engineering, University of Basrah \\ Basrah, Iraq \\ \{ghaida_alsuhail@yahoo.com
}

\begin{abstract}
In this paper, we develop an analytical energy efficiency model using dual switched branch diversity receiver in wireless sensor networks in fading environments. To adapt energy efficiency of sensor node to channel variations, the optimal packet length at the data link layer is considered. Within this model, the energy efficiency can be effectively improved for switch-and-stay combiner (SSC) receiver with optimal switching threshold. Moreover, to improve energy efficiency, we use error control of Bose-Chaudhuri-Hochquengh (BCH) coding for SSC-BPSK receiver node compared to one of non-diversity NCFSK receiver of sensor node. The results show that the $\mathrm{BCH}$ code for channel coding can improve the energy efficiency significantly for long link distance and various values of high energy consumptions over Rayleigh fading channel.
\end{abstract}

Keywords-BCH coding, Diversity, Energy efficiency, Sensor networks.

\section{INTRODUCTION}

The emergence of wireless sensor networks (WSNs) has currently become increased with wide-ranging applications from health, home, and environmental to military, space and commercial. They are a special case of ad-hoc wireless networks where the constraints on resources are especially tight [1]. WSNs are composed of nodes typically powered by batteries, for which replacement or recharging is very difficult. With finite energy, a finite amount of information can only be transmitted. Therefore, minimizing the energy consumption for data transmission becomes one of important design considerations for WSN in most application scenarios. Moreover, the channel fading has also a great effect on the reliability of data transmission and energy consumption in WSN. As a result, the design of energy efficient strategies to prolong lifetime or minimize the energy consumption is still of utmost and critical importance issue in WSN design [2][3].

More specifically, the distance between neighbour nodes in wireless sensor networks; such as IEEE 802.15./Zigbee; is shorter compared to other wireless networks like Wi-Fi. To make the best use of the limited energy available to the sensor

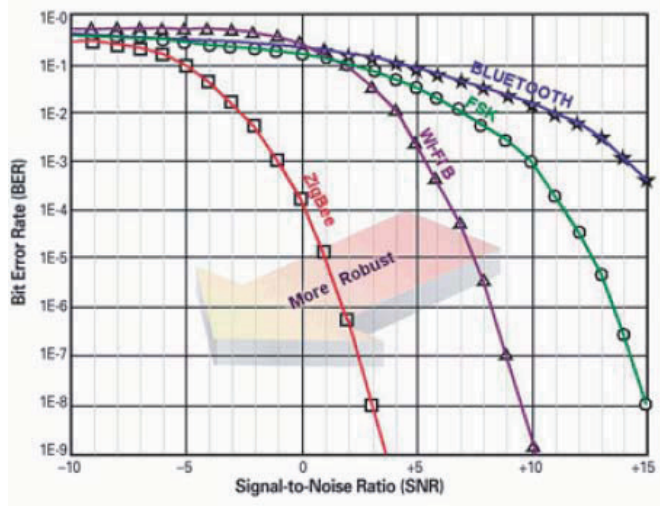

Fig. 1 BER vs. SNR [6]

nodes, and hence to the network, it is important to appropriately set parameters of the protocols in the network stack. Here, we specifically focus on the physical layer, where the parameters open to the network designer include: modulation scheme, transmit power and hop or link distance. The optimal values of these parameters will depend on the channel model such as AWGN and Rayleigh fading channels. When a wireless transmission is received, it can be decoded with certain probability of bit error (BER), based on the ratio of signal power-to- noise power of the channel (i.e., the SNR). As the energy used in transmission increases, the probability of error goes down, and thus the number of retransmissions goes down. Thus there exists an optimal trade-off between the expected number of retransmissions and the transmit power to minimize the total energy dissipated to receive the data [4]. For example, IEEE 802.15.4/Zigbee relies on a very robust modulation technique known as Phase Shift Keying (PSK), instead of FrequencyShift Keying (FSK). FSK is a less efficient, but simpler to implement and is used in a typical low-power, short range RFM-TR1000 transceiver (MICA motes) [5], Bluetooth and 
many other applications. Specifically, the both forms of BPSK and QPSK can provide $7 \mathrm{~dB}$ to $18 \mathrm{~dB}$ better BER performance, and it can be directly translated to a range that increases from 2 to 8 times the distance using the same energy per bit, or an exponential increase in reliability at any given range as illustrated in Fig. 1 [6].

On the other hand, to mitigate the channel fading effects a diversity technique is also powerful tool used in wireless communication systems [7]-[10]. The depth of the fades and/or the fade duration is reduced by supplying the receiver with multiple replicas of transmitted signal that have passed over independently fading channels. The simplest form of diversity combining schemes is classified as follows: (i) a classical non-switched diversity (NSD), i.e., selection combiner and (ii) Switched-and Stay combiner (SSC). Both combiners are SNR-based schemes. The SSC is an attempt to simplify the complexity of selection diversity system rather than continually connecting the diversity path with the best quality. The receiver selects a particular diversity path until its quality drops below a predetermined threshold. When this happens, the receiver switches to another diversity path [11]. In this paper, we have investigated the energy efficiency of SSC-BPSK receiver of sensor node under different energy consumptions of start-up, communication, and channel decoding for certain bit error rates conditions of Rayleigh fading channel.

\section{RELATED WORK}

Keeping in view of the energy limitations in WSNs, a large number of protocols and methods have been devoted for energy efficient communication. Many analytical energy consumption models [4][12] [13] have explored for single and multi-hop communication scenarios with and without the existing error control code (ECC) schemes and particular modulation scheme for WSN. In [12], they addressed optimal packet size for single hop using energy efficiency as the optimization metric, and $\mathrm{BCH}$ codes are found to be $15 \%$ more energy efficient than conventional codes.

In [13], different ECC techniques (Hamming, Golay, and $\mathrm{BCH})$ were investigated for single hop, and using Hybrid FEC/ARQ for multihop communication ignoring encoding/decoding energy consumptions and the impact of multi-use interference and start-up energy consumption. They showed the trade-off between the reliability of communication and the overhead of coding.

In [14], authors proposed a cross-layer energy detailed analysis of the radio transceiver energy consumption in physical layer, Medium Access Control (MAC) protocols namely non-persistent CSMA, S-MAC, and nanoMAC in data-link, and linear topology model in the network layer. Their results provided for single-hop communication up to $40 \%$ lower energy consumption than multi-hop communications. On the other hand, [15] have explored the energy-efficiency of various ECCs with field-programmable- gate-array (FPGA) and application-specific integrated-circuit (ASIC) implementations of encoder-decoder. The power consumption with ASIC implementation is an order of magnitude less than that with FPGA implementation. In addition, [16] have analyzed the distance at which an ECC becomes energy efficient for different environments and operating frequencies using different decoders implemented in ASIC. However, in commercial sensor nodes, low-power processors or microcontrollers are still used due to their versatility and cost effectiveness [1] in spite of encoding/decoding consumes an order of magnitude less power in ASICs. Processor-based studies [2] have proposed forward error correction (FEC) and ARQ for low-power design to calculate the energy based on the number of instructions executed for a particular encoding/decoding. The authors in [3] have measured the energy consumption of processor for Convolutional Codes (CCs) and found that such codes are not suitable for WSNs due to large decoding overheads. System-level methodology [17] has also been presented for exploring various ECCs with different modulation schemes (MPSK, MFSK and MQAM) taking into account the transmit signal energy, the circuit energy, the encoder energy and decoder energy as well. They found an energy-optimal ECC that reduces node energy consumption and in consequence increases the node life for different application-driven scenarios.

Choosing energy-efficient modulation scheme with lowcomplex implementation (referred to as green modulation), is a critical factor in the physical layer of WSNs. [18] presented the first in-depth analysis of energy efficiency of various modulation schemes such as $N C$-MFSK with small $M$ and $O O K$ that have significant energy saving compared to other schemes for short range scenarios. Both modulations have less complexity and less cost in implementation than the other schemes. They employed realistic models in IEEE 802.15.4 to find the best scheme in a proactive WSN over Rayliegh and Rician flat-fading channel models with path loss. The complexity of M-ary modulation schemes, for example MPSK, makes their implementation on WSNs rather costly despite great BER performance. They require Digital-toAnalog Converters (DAC) and mixers which are the most power intensive component at the receiver.

Furthermore, another energy saving techniques have also developed in the physical layer of WSNs. Like all other wireless networks, sensor networks suffer from the effects of fading. Therefore, multiple antenna communication (diversity) schemes in wireless systems are also available for WSNs in two ways as follows. (i) Multiple-Input MultipleOutput (MIMO)-based techniques such as space-time coding (STC) and smart antenna techniques [19]-[22] were proposed. However, a drawback is that they require complex transceiver circuitry and large amount of signal processing power resulting in large power consumptions at the circuit level. This fact has so far limited the application of MIMO techniques to WSNs because of limited battery of sensor 


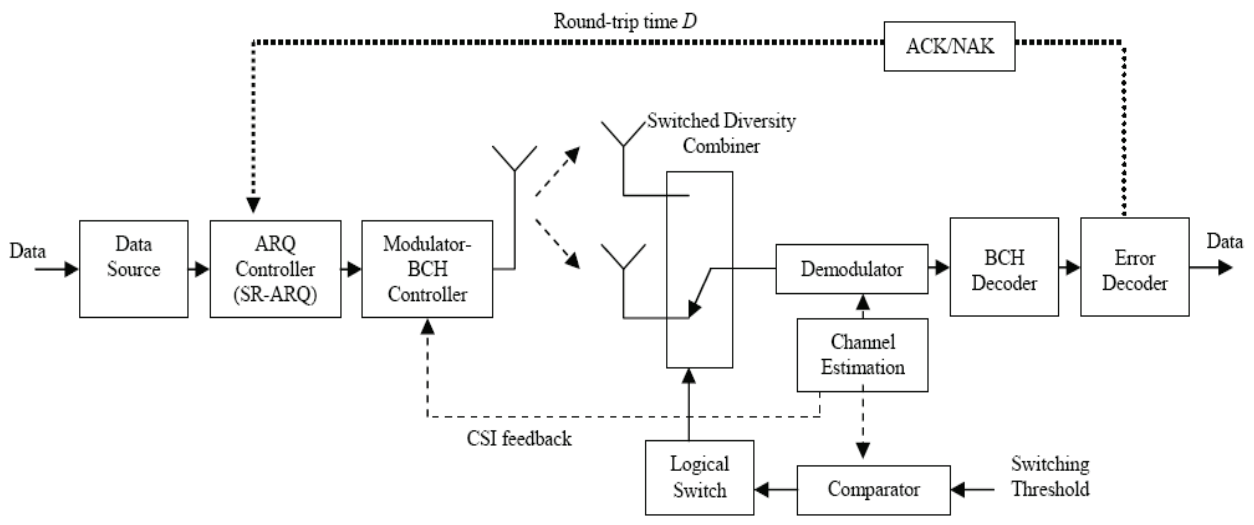

Fig. 2 A proposed block diagram of a switched dual selection diversity system for a single hop (point-to-point) in wireless sensor network.

nodes. (ii) Cooperative MIMO-based sensor networks (or Cooperative Diversity) may in fact result in better energy optimization where a group of sensors cooperate to transmit and receive data; this is also referred to as virtual MIMO networks. In fact, this way of diversity is used to mitigate the impact of fading through distributed antenna sharing. The form is suited towards WSNs because the size and power constraints restrict nodes to posse no more than one antenna. As a consequence, analysis of the resulting energy savings would be limited to 3 or 4 node networks [20]-[21].

In this paper, therefore, we propose an analytical energy model based on Switch-and-Stay Combiner (SSC) diversity receiver with optimal switching threshold in wireless sensor network. The energy efficiency metric of a single hop between two neighbour sensor nodes is examined at the radio data-link layer when SSC based coherent BPSK is employed instead of Non-coherent FSK scheme (NCFSK). To achieve this goal, we introduce the effect of retransmission using Selective-Repeat Automatic Repart request (SR-ARQ) [7][9], error control parities of $\mathrm{BCH}$ coding, and decoding energy on energy efficiency over Rayleigh fading channel. More precisely, the effect of start-up energy transient is investigated via the energy efficiency metric. This can be done by providing optimal packet size via pure ARQ scheme or adapting a reasonable error-correction capacity via a small number of $\mathrm{BCH}$ parity bits. The simulation results are compared to the NCFSK receiver of no-diversity antenna scheme.

\section{SYSTEM MODEL}

\section{A. SSC Diversity}

In this paper, we consider the receiver of wireless sensor consists of two antennas as shown in Fig. 2 where only one of which is used at a time for the space diversity reception system. The SSC diversity, i.e. pre-detection scheme, is an attempt to simplify the complexity of the non-switched selection diversity system rather than continually connecting the diversity path with the best quality. The receiver selects a particular diversity path until its quality drops below a predetermined threshold. When this happens, the receiver switches to another diversity path [8][10]. In other words, if a packet in radio-link/physical layer is received incorrectly, it is concluded that the current path has encountered a deep fade, and the receiver needs to select the other branch of SSC receiver.

At the transmitter, SR-ARQ controller and $\mathrm{BCH}$ controller are both considered to provide error protection on the transmitted data depending on the two feedbacks of CSI and ACK/NACK [7][9]. BCH controller is responsible for adding channel coding to packet (block) at the physical layer; and if the $\mathrm{BCH}$ decoder at the receiver fails in correcting the bit errors in the packet then SR-ARQ controller retransmits the entire packet once NACK signal is received. In Fig. 2, the wireless link implements the Selective Repeat (SR) protocol for retransmission of erroneous packets with perfect code detection (CRC) then suitably large buffers at the transmitter and the receiver are required.

\section{B. Channel Estimation}

In this section, we estimate the raw channel BER (Bit Error Rate) for typical wireless sensor networks. The probability of bit error is evaluated under a coherent BPSK modulated Rayliegh fading channel model for a dual-branch SSC diversity receiver in wireless sensor networks is defined as follows [11]:

$$
\begin{aligned}
p_{b, S S C}=\frac{1}{2}\left(1-e^{-\gamma_{T} / \gamma}\right) & \left(1-\sqrt{\frac{\gamma}{1+\gamma}}\right)+e^{-\gamma_{T} / \gamma} Q\left(\sqrt{2 \gamma_{T}}\right) \\
& -\sqrt{\frac{\gamma}{1+\gamma}} Q\left(\sqrt{2 \gamma_{T}(1+\gamma) / \gamma}\right)
\end{aligned}
$$

$\gamma: E_{b} / N_{o}$

$\gamma(d B)=77-10 \beta \log (d)[12]$ 


\section{$\beta:$ Path loss exponent $=4.0$}

where $\gamma$ is the average received bit energy to noise ratio per each receiver antenna branch. According to the receiver implementation, $\gamma$ depends on the neighbor distance $d$ between sensor nodes. The path loss is the average propagation loss as a function of the distance $d$ on the order of $\beta$.

$\gamma_{T}$ denotes the SNR switching threshold which leads to derive the optimal threshold value to minimize the average error rate of (7). Since the average BER is a continuous function of $\gamma_{T}$, there exists an optimal value of $\gamma_{T}$ for which the average BER is minimal. The optimum receiver threshold for BPSK transmission over Rayleigh fading can be obtained as

$$
\gamma_{T}^{*}=\frac{1}{2}\left[Q^{-1}\left(\frac{1}{2}\left(1-\sqrt{\frac{\gamma}{1+\gamma}}\right)\right)\right]^{2}
$$

The closed-form expression of (3) will allow us to compute only the optimum switching threshold $\gamma_{T}^{*}$ (in the minimum error rate sense) for identical fading statistics.

Fig. 3 presents the probability of bit error by (1) according to the neighbor distance compared to NC-FSK modulation scheme [23] with path loss exponent $\beta=4$ (or $\beta=3.5$ ) in the worst case design [11],

$$
p_{b, F S K}=\frac{1}{2+\gamma}
$$

For typical neighbor distances of 20-30 meters (indoor range) in WSN, we notice that the raw BER ranges between $7 \times 10^{-4}$ and $3 \times 10^{-3}$ in case of NC-FSK [12]; and of 60-75 meters (outdoor range) in case of SSC receiver based coherent BPSK defined in (1).

\section{PROPOSED ANALYSIS}

\section{A. Energy Consumption Characteristics}

The data packet in radio link layer consists of a header field of $h$ bits (few bytes), payload of size $L$ and a $\tau$ bits trailer (for parity bits for error control FEC purpose). In general, the header field includes the current segment number, total number of segments in the corresponding higher layer packet, higher layer packet identifier and the source and destination identifiers. Using this packet format and the energy model in [3], the energy required to communicate (transmit and receive) one bit of information (Eb) across a single hop is expressed as,

$$
E_{b}=E_{T}+E_{R}+\frac{E_{d e c}}{L}
$$

where,

$E_{\text {dec }}$ : decoding energy per packet (for BCH FEC purpose).

$E_{T}, E_{R}:$ Transmitter and Receiver Energy Consumptions per one bit

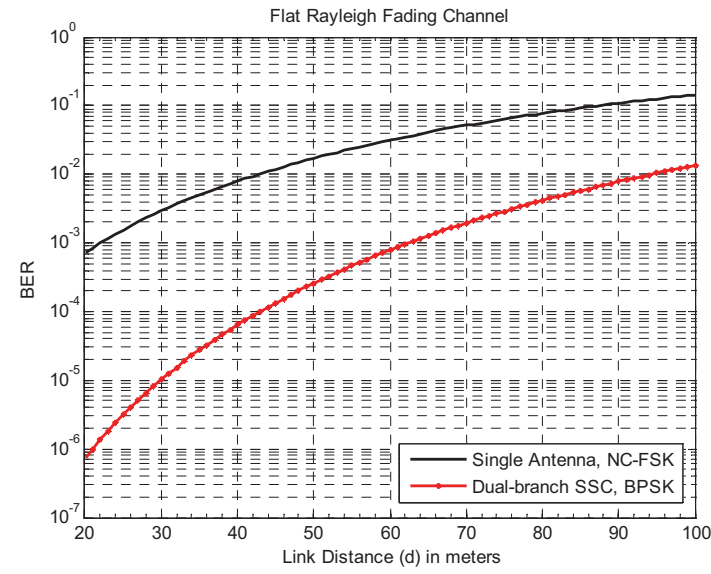

Fig. 3 The probability of bit error vs. link distance for two modulation schemes over Flat Rayleigh fading channel in WSNs.

Assuming the energy required for encoding process in transmitting a packet (or bit) is small to be ignored when FEC scheme is used, and

$E_{T}=\left(\left(P_{T e}+P_{\text {out }}\right) \frac{(L+h+\tau)}{R_{o}}+P_{T s t} T_{T s t}\right) \cdot L^{-1}$

$E_{R}=\left(P_{\operatorname{Re}} \frac{(L+h+\tau)}{R_{o}}+P_{R s t} T_{R s t}\right) \cdot L^{-1}$,

where:

$P_{T e}, P_{\mathrm{Re}}: \quad$ Power consumed in Transmitter/Receiver electronics.

$P_{T s t}, P_{R s t}:$ Start-up power consumed in Tx/Rx.

$T_{T s t}, T_{R s t}:$ Transmitter/Receiver start-up time.

$P_{\text {out }}$ : Output Transmit Power. (e.g., $-24 \mathrm{dBm}$ to $0 \mathrm{dBm}$ ).

$R_{o}$ : Data rate (e.g., $\approx 20 \mathrm{Kbps}$ in MICA2 mote) [5].

Rewriting (5) in terms of radio parameters $k_{1}$ and $k_{2}$, then the energy consumption per one bit can be expressed,

$E_{b}=k_{1}+k_{2} \frac{(h+\tau)}{L}+\frac{k_{2}+E_{d e c}}{L}$

To simplify the manipulation, $k_{1}$ and $k_{2}$ are defined in terms of $E_{c}$ and $E_{s}$ as follows

$$
\begin{gathered}
E_{c}=k_{1}=\frac{\left(P_{T e}+P_{o u t}\right)+P_{\mathrm{Re}}}{R_{o}} \\
E_{s}=k_{2}=\left(P_{T s t} T_{T s t}+P_{R s t} T_{R s t}\right)
\end{gathered}
$$

$k_{1}\left(E_{c}\right)$ and $k_{2}\left(E_{s}\right)$ are constants for a given radio transceiver and $R_{o}$ is being a data rate. $k_{1}$ defines the useful energy in the communication of a bit and $k_{2}$ defines the start-up energy 
consumption. In addition, from (5), $E_{b}$ is inversely proportional to the payload $L$ for given values of $h$ and $\tau$ and assuming the term $\left(E_{d e c} / L\right)$ remains fairly constant. In this paper, we will consider the same parameter values in MICA motes [12]: $k_{1}=1.85 \mu \mathrm{J} / \mathrm{bit}$ and $k_{2}=24.86 \mu \mathrm{J}$ to demonstrate some reasonable comparison in our simulation when coherent BPSK of SSC scheme is employed.

\section{B. Power Management and Optimal Packet Length}

Power management is defined by turning off the transceiver sensor to reduce energy consumption when it is in the idle state. With the use of power management, it is possible to accomplish significant energy savings. In fact, although sensor nodes normally use short packets in communication, the dominance of stat-up energy is still a key challenge in reducing energy efficiency. The energy efficiency, in consequence, can be expressed as,

$$
\begin{aligned}
& \eta_{e}=\zeta_{\text {th }} \cdot R \\
& \eta_{e}: \text { Energy efficiency } \zeta_{\text {th }}: \text { Energy throughput } \\
& R: \text { Reliability }
\end{aligned}
$$

where $\zeta_{t h}$ represents the ratio of energy consumed for actual data transmission to entire packet transmission, and $R$ denotes the successful packet reception rate (1-PER).

To provide power management as a function of only packet length without error control (i.e., $E_{d e c}=0$ ), (5) can be rewritten to explain energy efficiency when start-up energy consumption $(E s)$ plus communication energy consumption $\left(E_{c}\right)$ are taken into a consideration as follows:

$$
\eta_{e}=\frac{E_{c} L}{E_{c}(L+h)+E_{s}}(1-P E R)
$$

\section{$L:$ payload length \\ $h$ : header length \\ PER $=(1-p)^{(L+h)}$ : packet error rate}

To maximize $\eta_{e}$ with respect to the payload length $L$, it can be shown that there exists a unique maximum value for the optimization function in (10). The optimal payload length without coding can be obtained by differentiating (10) to $L$ and setting it to zero,

$$
L_{n o, c o}^{*}=\frac{1}{2}\left(\sqrt{C_{o}^{2}-4 C_{o} / \ln (1-p)}-C_{o}\right)
$$

with $C_{o}=h+k_{2} / k_{1}$ (or $h+E_{s} / E_{c}$ ), where $C_{o}$ represents the energy consumption coefficient used for a power management in each transceiver sensor node. The metric $p$ is the raw channel BER and can be obtained from (1) or (4) depends on modulation scheme used over the wireless channel. In practice, $L_{n o, c o}^{*}$ is usually rounded off to the nearest byte.

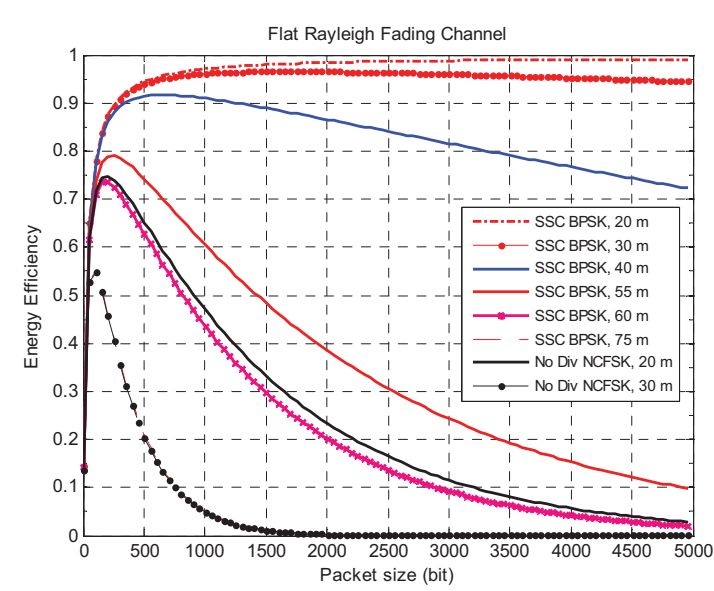

Fig. 4 The energy efficiency as a function of payload size when no error control is used over Rayleigh fading channel. Assume $h=16$ bits, $E c=k_{1}=1.85 \mu \mathrm{J} / \mathrm{bit}$ and $E s=k_{2}=24.86 \mu \mathrm{J}$.

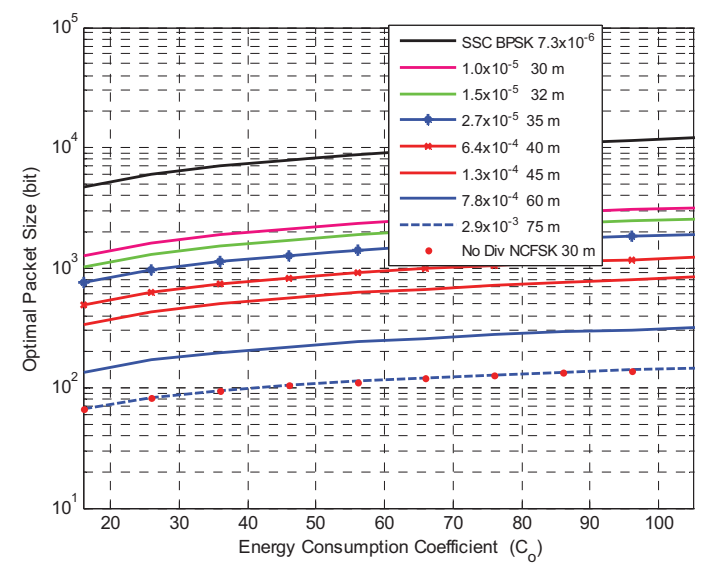

Fig. 5 The energy consumption coefficient vs. optimal payload size when no error control is used over Rayleigh fading channel. Assume $h=16$ bits.

Fig. 4 demonstrates the energy efficiency against the packet size without error control for various values of link distance (or the corresponding raw channel bit error rate $p$ ). It is clearly noticed that optimal packet size can be achievable when maximum energy efficiency is obtained with decreasing $p$ over the channel. The smaller packet size which is less than optimal values introduces a dramatic decrease in efficiency and this behaviour is referred to the higher overhead and start-up energy consumed for these small packets. In addition, the larger payload size which is greater than optimal size can also slowly drop the efficiency but with much channel reliability $(R)$ for long distances (i.e., lower values of p). 
At lower values of distance $(20 \mathrm{~m}-30 \mathrm{~m})$, i.e. lower region values of $p$, SSC receiver of BPSK modulation outperforms the one of non-diversity NCFSK receiver. The efficiency curve in this case almost attains a flat top for packet size greater than 500 bits. Also, it is shown that the lowest value of maximum achievable energy efficiency can be $54.84 \%$ for $p=3 \times 10^{-3}$ in case of SSC-BPSK scheme with $d=75 \mathrm{~m}$ and non-diversity NCFSK with $d=30 \mathrm{~m}$.

On the other hand, Fig. 5 depicts the obtained optimal packet size which effectively depends on two parameters of $p$ and $C_{o}$ over Rayleigh fading channel. For reasonable range of radio parameters of $k_{1}$ and $k_{2}\left(E_{c}\right.$ and $\left.E_{s}\right)$ an optimal packet size can be obtained by evaluating the energy consumption coefficient and the channel bit error rate and the corresponding link neighbor distance.

As a consequence, in this paper, to improve this energy efficiency curves our focus will be only on the dramatic drop (raising or falling) in the curves; and hence the optimal payload size will be limited to be no larger than 512 bits when $\mathrm{BCH}$ error control is applied in the radio link layer as explained in Section IV-C.

\section{Power Management and BCH Coding}

A channel coding is required for wireless communications to protect data from the errors which may result from noise, fading, and interference. For low SNR region of spread spectrum modulation, where each bit is multiplied by a chip sequence and spread into $n$ bit times, or time division multiplexing, a common idea of non-extending a period time of the block (packet) can be applied. This does not increase the energy per information bit, and such variation is called namely an adaptive FEC.

We now consider binary $\mathrm{BCH}$ codes with hard decision where the encoder adds a number of $\tau$ parity bits to the $L$ payload and $h$ header. As a result, the packet length $n$ equals $L+h+\tau$. The energy efficiency of the $\mathrm{BCH}$ code with the $t$ error correction capability is expressed as,

$\eta_{S S C, e c}=\left(\frac{E_{L}}{E_{\text {Total }}}\right) \times R_{e c}=\xi_{e c} \times R_{e c}$

where $R_{e c}$ and $\xi_{e c}$ represent reliability of error control and energy throughput of encoded block (packet), respectively. $E_{L}$ is being communication energy consumption of encoded payload length and $E_{\text {Total }}$ denotes total energy consumption of encoded packet communication, start-up energy consumption, and decoding energy. Hence,

$R_{e c}=\left(1-P E R_{e c}\right)=\sum_{i=0}^{t}\left(\begin{array}{l}n \\ i\end{array}\right) p_{b}^{i}\left(1-p_{b}\right)^{n-i}$

$E_{L}=E_{c}(n-h-\tau)$
$E_{\text {Total }}=E_{c} n+E_{s}+E_{\text {dec }}$

$n$ is assumed variable parameter but not exceeding a maximum limit $n_{\max }$. For numerical results, a typical $n$ is considered to equal up to 512 bits. Moreover, the decoding energy $E_{d e c}$ defined for a $t$ error correction binary BCH code can be expressed as,

$E_{d e c}=\left(2 n t+2 t^{2}\right)\left(E_{\text {add }}+E_{\text {mult }}\right)$

where $E_{\text {add }}$ and $E_{\text {mult }}$ are the energy consumption in addition and multiplication under $\mathrm{GF}\left(2^{m}\right), m=\left|\log _{2} n+1\right|$. For example, $E_{\text {add }}=3.3 \times 10^{-5} \mathrm{~m} \quad(\mathrm{~mW} / \mathrm{MHz})$ and $E_{\text {mult }}=3.7 \times 10^{-5} \mathrm{~m}^{3}$ $(\mathrm{mW} / \mathrm{MHz})$ [5]. Now, the energy throughput in (12) can be expressed again as follows [23],

$\xi_{e c}=\left(\frac{n-h-\tau}{n-h+C_{0}+C_{1}}\right)$

$C_{l}=E_{\text {ded }} / E_{c}$ represents energy coefficient in the presence of $\mathrm{BCH}$ coding, then the optimal energy efficiency can be achieved,

$$
\begin{aligned}
L_{e c}^{*}, \tau^{*}, P E R_{e c}^{*}= & \arg \max \eta_{s S C, e c}\left(L_{e c}, t, P E R_{e c}\right) \\
& L_{e c} \in n, \tau \in C_{b o u n d}, P E R_{e c} \in P E R
\end{aligned}
$$

$C_{\text {bound }} \leq m \times t$ denotes the bound of inclusive period of total parity bits per block. When $C_{\text {bound }}=m \times t$, then $\tau=m \times t$ becomes an indicator of the worst performance of $\mathrm{BCH}$ in terms of energy efficiency. This indicator also defines the lower bound of $\mathrm{BCH}$ code, i.e. the maximum number of parity bits required for a certain value of error correction capacity $(t)$.

\section{Performance Evaluation}

In this section, the results were conducted on SSC scheme performance using two error control schemes: (i) a pure ARQ and (ii) ARQ combined with error control of $\mathrm{BCH}$ coding called a hybrid ARQ Type I. Specifically, we examine the effect of the redundant amount of $\mathrm{BCH}$ channel coding to provide the maximal energy efficiency over a flat Rayleigh fading channel.

First, we investigate the effect of $\mathrm{C}_{1}$ (decoding energy w.r.t. the communication energy) on the energy efficiency for different link distance when SSC scheme based BPSK is considered in the receiver side. In Fig. 5 a, it is noticed that the error control is not essential to be added to packet when link distance is less than $60 \mathrm{~m}$ because the energy efficiency degrades for any few redundancy parity bits. Meanwhile, Fig. 5 (b) depicts a clear effect of $\mathrm{BCH}$ parity bits and one or two error correction bits can be achievable to improve the energy 
efficiency over the variable energy coefficients of start-up and communication states. As a result, for link distance equal or greater than $60 \mathrm{~m}$, we can conclude that the error control has a significant effect on the energy efficiency but only for small $\mathrm{BCH}$ codes with $t=1$ or $t=2$. On the other hand, halving the ratio of decoding energy w.r.t. communication energy introduces another clear increment in the energy efficiency especially for a small $\mathrm{BCH}$ codes. Furthermore, it is noticed that with $t=2$ the energy required for addition and multiplication processes at the $\mathrm{BCH}$ decoder will also reduce to the half in both cases of Fig. 5 (a) and (b) using (12).

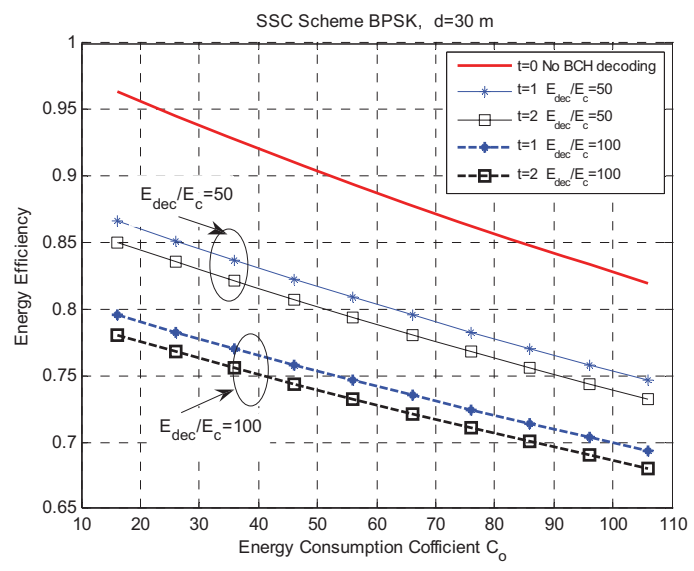

(a)

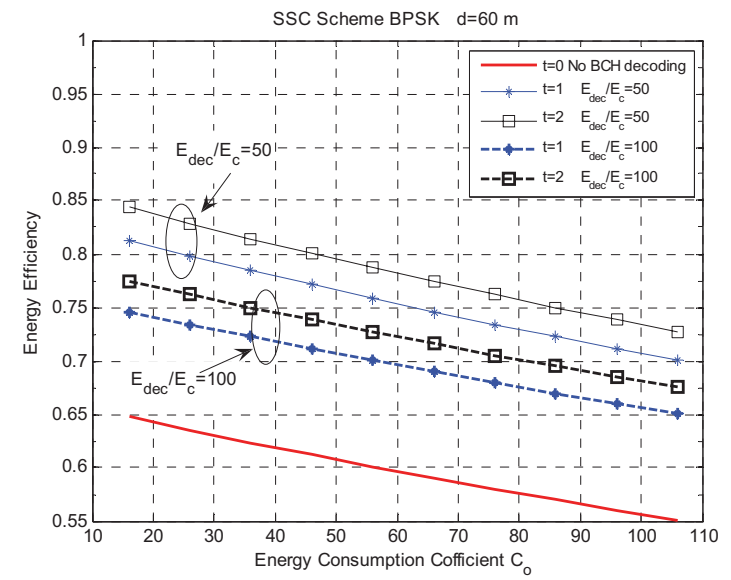

(b)

Fig. 5 The Effect of $\mathrm{BCH}$ error correction bits on energy efficiency over Rayleigh fading channel. Assume $n=511$ bits, $h=16$ bits in SSC scheme. (a) $d=30 \mathrm{~m}$ and (b) $d=60 \mathrm{~m}$

Fig. 6 displays another comparison for the effect of $\mathrm{BCH}$ error coding on the energy efficiency when non-diversity NCFSK receiver is used. We consider fixed $\mathrm{n}$ of 511 bits, $\mathrm{h}$ of 16 bits and $\mathrm{d}$ of no more than 30 meter in order to provide a reasonable link BER (reliability) for communication. It is clearly shown that the significant difference in the efficiency performance compared to results in Fig. 5a (SSC diversity receiver). The efficiency is improved to be nearly $70 \%$ at ideal system (i.e., no power or energy management) and once the energy consumption coefficient $\left(\mathrm{C}_{\mathrm{o}}\right)$ increases the overall system efficiency will slowly and linearly degrade and result small variation in efficiency of no more than $5 \%$ for all curves.

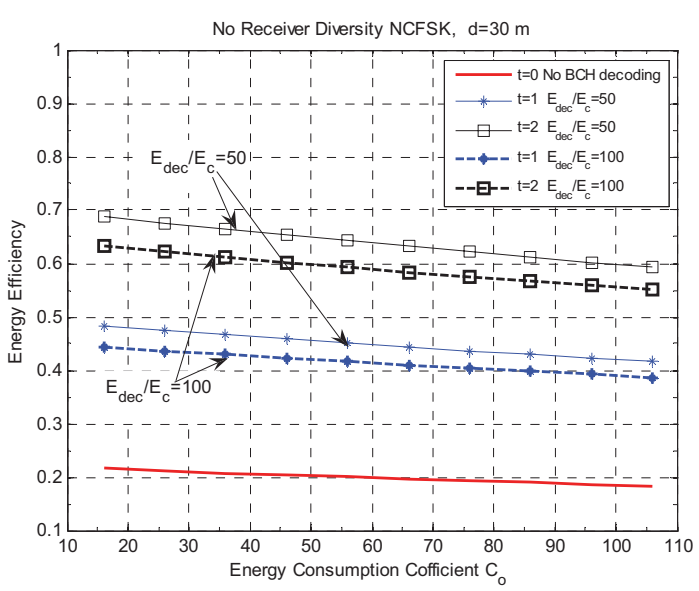

Fig. 6 The effect of $\mathrm{BCH}$ error correction bits on energy efficiency over Rayleigh fading channel. Assume fixed $n=511 \mathrm{bits}, h=16$ bits in no-diversity NCFSK receiver with $d=30 \mathrm{~m}$.

\section{CONCLUSION}

This paper has dealt with an analytical energy efficiency model based on a dual switched branch diversity receiver in wireless sensor networks. A channel model is considered as a flat Rayleigh fading for both non-diversity NCFSK receiver and our proposed dual-switched branch receiver of coherent BPSK. To adapt energy efficiency of sensor node to channel variations, the optimal packet length is applied. Furthermore, it is noticed that the energy efficiency of SSC diversity scheme with optimal switching threshold outperforms the non-diversity NCFSK especially for link distance greater than 60 meter. The later scheme was limited by its bad link BER as long as the neighbour distance increases. On the other hand, the results clearly showed that when small $\mathrm{BCH}$ codes are applied for both receivers, SSC scheme provides a significant gain in the energy efficiency under the constraint of the increase in the energy consumption coefficient $\mathrm{C}_{0}$ (energy of start-up to energy of communication) for a single hop (i.e., point-to-point link). For future work, the analytical energy model in this paper can be extended to involve cooperative diversity in the linear multi-hop topology in WSNs using different antenna diversity scheme in order to enhance the energy efficiency of the network. 


\section{REFERENCES}

[1] M. Vieira, C.N. Coelho, D.C. da Silva, "Survey on Wireless Sensor Network Devices," IEEE Conf. Emerging Technol. Factory Autom., pp. 537-544, Sep. 2003.

[2] P. Lettieri, C. Fragouli, and M.B. Srivastava, "Low Power Error Control for Wireless Links," ACM/IEEE Int. Conf. Mobile Comput. Netw., pp. 139-150, 1997.

[3] E. Shih, S. H. Cho, F. S. Lee, B. H. Calhoum, and A. Chandrakasan, "Design Considerations for Energy-Efficient Radios in Wireless Microsensor Networks," J. VLSI Signal Process, vol. 37, no. 1, pp. 77 94, May 2004.

[4] M. Holland, Optimization Physical Layer Parameters for Wireless Sensor Networks, MSc. Thesis in Electrical and Computer Engineering, University of Rochester, New York, 2007.

[5] M. Goel and N. R. Shanbhag, "Low Power Channel Coding Via Dynamic Reconfiguration," Proceedings of ICASSP, Phoenix, AZ, pp. 1893-1896, March 1999.

[6] http: \\www.embeddedcomputing.com

[7] S. S. Chakraborty, M. Liinaharja and K. Ruttik, "Diversity and packet combining in Rayleigh fading channels," IEE Proc. Commun, vol. 152, no.3, pp. 353-356, June 2005.

[8] X. Cai and G. B. Giannakis, "Performance Analysis of Combined Transmit Selection Diversity and Receive Generalized Selection Combining in Rayleigh Fading Channels," IEEE Trans on Wireless Commun., 2004.

[9] E. Cianca, A. D. Luise, M Ruggieri, and R. Prasad "Channel-Adaptive Techniques in Wireless Communications: An Overview." Wireless Commun. Mob. Compu., 2:799-813, 2002.

[10] B. Holter and G. E. Oien, "Performance Analysis of a Rate-Adaptive Dual-Branch Switched Diversity System," IEEE on Trans. on Commun., vol. 56, no. 12, pp. 1998-2001, Dec. 2008.

[11] M. K. Simon and M.-S. Alouini, Digital Communication over Fading Channels, $2^{\text {nd }}$ edition, J. Wiley, New Jersey, 2005.

[12] Y. Sankarasubramaniam, I. F. Akyildiz and S. W. McLaughlin, "Energy Efficiency based Packet Size Optimization in Wireless Sensor Networks", Proc. of the $1^{\text {st }}$ IEEE International Workshop on Sensor Network Protocols and Applications, May 2003.
[13] H. Karvonen, C. Pomalaza-Raez and M.Hamalainen, "Cross-Layer Energy Efficiency of FEC Coding in UWB Sensor Networks," Proc. of IEEE Inter. Conf. on Ultra-Wideband, pp. 337-362, Sept. 24-27 2006.

[14] J. Haapola, Z. Shelby, C. Pomalaza-Raez, and P. Mahonen, "Crosslayer Energy Analysis of Multip-hop Wireless Sensor Networks," IEEE Proceedings of the $2^{\text {nd }}$ European Workshop on Wireless Sensor Networks, Feb 2005

[15] G. Balakrishnan, M. Yang, Y. Jiang, and Y. Kim, "Performance Analysis of Error Control Codes for Wireless Sensor Networks," Int. Conf. Inf. Technol., pp. 876-879, 2007.

[16] S. L. Howard, C. Schlegel, and K. Iniewski, "Error Control Coding in Low-power Wireless Sensor Networks: When is ECC energyefficient?," EURASIP J. Wireless Commun. Netw., no. 2, pp. 1-14, 2006.

[17] S. Chauhan, R. Bose, and M. Balakrishnan, "A Framework of EnergyConsumption-Based Design Space Exploration for Wireless Sensor Nodes," IEEE Trans. On Computer-Aided Design of Integrated Circuit and system, vol. 38, no. 7, July 2009

[18] J. Abouei, K.N. Plataniotis, and S. Pasupathy, ,"Green Modulation in dense Wireless Sensor Networks," IEEE Inter. Conference on Acoustic, Speech and Signal Processing (ICASSP'09), Sept. 2009.

[19] S. K. Jayaweera, "Energy Analysis of MIMO Techniques in WSNs, Proc. Annu. Conf. on Info. Sciences and Systems (CISS), USA, Princeton, March 2004.

[20] S. Hussain, A. Azim, and J. H. Park, "Energy Efficient Virtual MIMO Communication for Wireless Sensor Networks," Springer, Telecommun Syst (2009): 42:139-149, June 2009

[21] N. Shastry and R.S. Adve, "A Theoretical Analysis of Cooperative Diversity in Wireless Sensor Networks," GlobCom05, 2005.

[22] S. Wang and J. Nie, "Energy Efficiency Optimization of Cooperative Communication in Wireless Sensor Networks," EURASIP J. on Wireless Comm. And Networking, vol. 2010.

[23] I. Joe, "Optimal Packet Length with Energy Efficiency for Wireless Sensor Networks," IEEE Inter. Symp. Circ. And Sys. ISCAS2005, 2320 May 2005. 